\title{
Modeling Regulatory Networks at Virginia Tech
}

\author{
NICHOLAS A. ALLEN, ${ }^{1}$ LAURENCE CALZONE, ${ }^{2}$ KATHERINE C. CHEN, ${ }^{2}$ \\ ANDREA CILIBERTO ${ }^{2}$ NAREN RAMAKRISHNAN, ${ }^{1}$ CLIFFORD A. SHAFFER, \\ JILL C. SIBLE, ${ }^{2}$ JOHN J. TYSON, ${ }^{2}$ MARC T. VASS, ${ }^{1}$ LAYNE T. WATSON, \\ and JASON W. ZWOLAK ${ }^{1}$
}

\begin{abstract}
The life of a cell is governed by the physicochemical properties of a complex network of interacting macromolecules (primarily genes and proteins). Hence, a full scientific understanding of and rational engineering approach to cell physiology require accurate mathematical models of the spatial and temporal dynamics of these macromolecular assemblies, especially the networks involved in integrating signals and regulating cellular responses. The Virginia Tech Consortium is involved in three specific goals of DARPA's computational biology program (Bio-COMP): to create effective software tools for modeling gene-proteinmetabolite networks, to employ these tools in creating a new generation of realistic models, and to test and refine these models by well-conceived experimental studies. The special emphasis of this group is to understand the mechanisms of cell cycle control in eukaryotes (yeast cells and frog eggs). The software tools developed at Virginia Tech are designed to meet general requirements of modeling regulatory networks and are collected in a problem-solving environment called JigCell.
\end{abstract}

\section{INTRODUCTION}

T HE LIVING CELL is a miniature, membrane-bound, biochemical machine that harvests material and energy from its environment and uses them for maintenance, growth and reproduction. These processes are carried out by macromolecular machines (enzymes, ribosomes, transport proteins, structural proteins, motor proteins) whose structures are encoded in nucleotide sequences (DNA and mRNA). The activities of these macromolecules are controlled and coordinated by regulatory networks of great complexity and exquisite effectiveness. These networks collect information from inside and outside the cell, process the data, and direct cellular responses that foster the survival and reproduction of the cell (Bray, 1995). How these regulatory systems work is no more or less apparent from their network diagrams than is a complex piece of electronics from its schematic wiring diagram. Whereas electrical engineers create accurate mathematical representations of wiring diagrams and use these equations to design new devices, molecular biologists are not accustomed to this kind of approach. To employ quantitative modeling as a means for deeper scientific understanding and for more rational engineering of cellular responses, there needs to be a paradigm shift in the field.

The ${ }^{1}$ Department of Computer Science and ${ }^{2}$ Department of Biology, Virginia Polytechnic Institute and State University, Blacksburg, Virginia. 
To this end, DARPA's Bio-Computation Program is supporting development of BioSPICE (Simulation Program for Intra-Cellular Evaluation), a collection of interoperable programs for model development, simulation, analysis, and comparison to experimental data. The tools are developed by expert computer scientists, in close collaboration with experienced modelers, and used to build sophisticated models that accurately represent molecular control systems. The models are based on experimental results retrieved from data repositories, and specific predictions of the models are tested experimentally by molecular biologists within the program.

The Virginia Tech Consortium is led by experienced modelers (John Tyson and Kathy Chen of Virginia Tech, Bela Novak of the Budapest University of Technology and Economics), experimentalists (Jill Sible of Virginia Tech, Fred Cross of The Rockefeller University, and Michael Mendenhall of the University of Kentucky Medical School), and computer scientists (Cliff Shaffer, Layne Watson and Naren Ramakrishnan of Virginia Tech). The group's goals are to develop accurate models of cell growth and division (Table 1), to conduct novel experimental tests of some of these models (Table 2), and to create general-purpose software tools (Table 3). This paper reviews progress to date in these three areas. Technical details are provided in cited references and in papers in preparation.

\section{MODELING AND EXPERIMENTATION}

The cell division cycle, the sequence of events by which a cell replicates all its components and divides them equally between two daughter cells, is an ideal test case for the BioSPICE program. The cell cycle is a regulatory system of fundamental biological significance, governed (in eukaryotes) by a universal mechanism that has been characterized in great detail both genetically and biochemically (Murray and Hunt, 1993). Realistic and accurate models are available, which make specific predictions that can be tested experimentally. However, cell cycle modeling has now reached the limit of what can be "hand-crafted," and the next level of computer simulation will require the type of tools envisaged by BioSPICE.

Our modeling efforts center around two specific experimental systems: frog eggs and budding yeast cells. Frog eggs provide a convenient system for biochemical studies, especially in cytoplasmic extracts (Murray and Kirschner, 1989). By supplementing egg extracts with recombinant proteins, one can manipulate the regulatory network to almost any specifications (Kumagai and Dunphy, 1995; Solomon et al., 1990). Budding yeast is an ideal organism for genetic characterization of molecular regulatory systems, and most of the genes encoding its cell cycle control system are now known (Mendenhall and Hodge, 1998).

\section{Frog eggs and extracts}

Regulation of Cdk1-cyclin B activity: parameter estimation. Ten years ago, Novak and Tyson (1993) published a thorough theoretical study of the mechanism of mitotic control in frog eggs (Xenopus laevis).

Table 1. Survey of Models

\footnotetext{
Cell division cycle

Budding yeast-molecular regulation of "Start" (G1-to-S transition) and "Finish" (metaphase-anaphase-telophase transitions); mechanism of the morphogenetic checkpoint (impaired bud formation)

Fission yeast-comprehensive model of G1-S, G2-M and meta-anaphase transitions; septation initiation network

Frog egg — patterns of cyclin-dependent kinase activation in early embryonic development (Cdk1-cyclin B activity and Cdk2-cyclin E activity); regulation of Cdk1-cyclin B activity in frog egg extracts

Other phenomena

Growth patterns in fission yeast-spatiotemporal organization of zones of actin polymerization, which determine regions of cell wall extension and hence cell shape

Circadian rhythms in fruit flies-oscillations and steady states in models of the synthesis and degradation of PERIOD and TIMELESS proteins in fly brain cells; mechanism of robust temperature compensation

Apoptosis (programmed cell death)—autocatalytic activation of caspases; mechanisms to stabilize the inactive state; irreversible activation
} 
Table 2. Critical Experimental Tests of Cell Cycle Models

Budding yeast

Nuclear localization of Cln-type cyclins controls cell size at division (Miller and Cross, 2001)

Bistability of the control system (Cross et al., 2002)

Two pathways for exit from mitosis (Cross, 2003)

Fission yeast

Branching patterns in cells that are defective in microtubule polymerization (under investigation in Paul Nurse's laboratory)

Frog egg

Rate constants for phosphorylation and dephosphorylation reactions (Kumagai and Dunphy, 1995; Lee et al., 1994)

Bistability of the Cdk-1-cyclin B activation system (Pomerening et al., 2003; Sha et al., 2003)

Critical slowing down close to the activation threshold (Sha et al., 2003)

Activation threshold raised by agents that block DNA synthesis (Sha et al., 2003)

A simplified version of their model is presented in Figure 1 as a graph, where labeled directed edges represent chemical reactions transforming substrates into products (the vertices of the graph). Reactions cause the concentrations, $C_{i}(i=1, \ldots, N)$, of the chemical species (the substrates and products) to change in time according to a system of $N$ ordinary differential equations $\frac{d C_{i}}{d t}=\sum_{j=1}^{R} b_{i j} v_{j}(i=1, \ldots, N)$, where $v_{j}$ is the velocity of the $j$ th reaction in the network and $b_{i j}$ is the stoichiometric coefficient of species $i$ in reaction $j\left(b_{i j}<0\right.$ for substrates, $>0$ for products, and $=0$ for species that are not transformed by the reaction). The velocities are nonlinear functions of the "state variables," $C_{i}$, and of "parameters" (rate constants, binding constants, enzyme concentrations). When the number of molecules per cell is small, as is often the case in cell biology, the governing equations are stochastic differential equations. The full set of rate equations is a mathematical representation of the temporal behavior of the regulatory network. Modelers are faced with many computational problems: accurately and efficiently solving equations when velocities vary over many orders of magnitude, finding steady state solutions (or distributions), estimating rate constants by fitting numerical solutions to experimental data, and identifying bifurcation points in the multi-dimensional parameter space.

Table 3. Overview of Software Tools

JigCell components

Model Builder-assists users in specifying a reaction network and deriving its governing kinetic equations

Run Manager—organizes numerical input to the kinetic equations, necessary for simulating specific experiments

Comparator-organizes experimental data, associates data to model simulations, computes a score reflecting the goodness-of-fit of the model to the data

Working components not yet incorporated into JigCell

Parameter Estimator - determines optimal parameter values for a model by minimizing the "distance" between simulations and experimental data; uses Levenberg-Marquardt method (based on local gradients)

VT DIRECT - determines the global optimum of the fitting problem by an efficient and rational search within a region of parameter space

Steady State Finder - computes all steady state solutions of an elementary chemical reaction network (mass action rate laws only); uses a globally convergent homotopy method implemented in the software package

POLYSYS_PLP (Wise et al., 2000)

Components under construction

Utilities-Dashboard, Installer, Tutorials, Help Facility

Database Managers_for models, simulations, comparisons, optimizations

Compare ${ }^{2}$ - tools for comparison of alternative models

Numerical Bifurcation Analysis-seamless access to the best freeware; database management to store, organize, retrieve, and search results 
For example, Novak and Tyson (1993), in order to match their model to the known properties of DNA synthesis and nuclear division in intact eggs and extracts, had to estimate numerical values of dozens of kinetic constants that appeared in their equations. Although there were, at the time, direct experimental measurements of only one of these constants (for cyclin degradation), Novak and Tyson were able to predict rate constants for the crucial phosphorylation and dephosphorylation reactions simply by fitting their model to basic qualitative facts about the control system. A few years later, Kumagai and Dunphy (1995) and Lee et al. (1994) observed the rates of these reactions on recombinant proteins in egg extracts, obtaining values in close agreement with theoretical predictions (Marlovits et al., 1998). An early goal of the Virginia Tech consortium was to use automatic parameter estimation to find an optimal fit of the Novak-Tyson model to the data summarized in Marlovits et al. (1998).

Optimal parameter values are determined by fitting solutions of the reaction kinetic equations to a suite of experimental observations. The experiments are usually of diverse types: time courses of individual components, phenotypes of mutants, thresholds for response. They come from different laboratories, under subtly different conditions, on different cell types. The "weight" given to each measurement is often a subjective decision. Measurements are rarely repeated enough times to generate statistically reliable estimates, and measurement errors in the independent variable are often comparable to errors in the dependent variable. In such cases, we propose to use an algorithm, ODRPACK, that constructs the orthogonal distance between the data and the model and minimizes this "objective function" by the Levenberg-Marquardt method. The kinetic equations of the model are solved by LSODAR. This approach applies quite generally to optimization problems in the context of biochemical regulatory circuits.

Using this approach, Zwolak et al. (2003) reconsidered the parameter estimation problem described by Marlovits et al. (1998). Taking Marlovits' informal parameter estimates as initial values, Zwolak found that ODRPACK quickly converged to a locally optimal parameter set close by, and the regression error was significantly improved. Zwolak also characterized the basin of attraction near this optimal solution. Zwolak's work is only a first step toward the goal of meaningful parameter estimation in cell cycle models. The team is actively pursuing both local and global optimization strategies, as well as parameter survey tools like Latin hypercube sampling.

Regulation of Cdk1-cyclin B activity: experimental confirmation of bistability. The original paper by Novak and Tyson (1993) made some striking predictions about the activation of MPF (M-phase promoting factor, the active form of Cdk1-cyclin B) (Fig. 1). Frog egg extracts, as prepared by Solomon et al. (1990), have copious amounts of Cdk1 but no cyclin B. By adding fixed amounts of recombinant, non-degradable cyclin B, Solomon observed a distinct threshold below which MPF activity is negligible and above which it is proportional to the total amount of cyclin B in the preparation (Fig. 2a). The Novak-Tyson model accounts for this threshold in terms of a saddle-node bifurcation in the kinetic equations and makes three predictions about the threshold (Fig. 2b):

(i) There should be a different (lower) threshold for MPF inactivation, when cyclin B concentration is decreased in an initially active extract. This result would indicate that mitotic entry and exit are regulated by a hysteresis loop.

(ii) The time delay for MPF activation is inversely proportional to cyclin B concentration when the concentration is just above the threshold. The data of Solomon et al. (1990) did not support this prediction, and a more quantitative re-examination of the timing of MPF activation was called for.

(iii) The threshold value of cyclin B should increase in the presence of inhibitors of DNA synthesis. This prediction concerns the fundamental control system regulating cell cycle checkpoints, which arrest progression through the cell cycle when genomic integrity is threatened.

In a recently published paper (Sha et al., 2003), Sible and her coworkers report confirmation of all three predictions (Fig. 2c,d). Prediction (i) was also confirmed independently by Pomerening et al. (2003). Sha's results, as an example of bridging experimental and theoretical work, were prominently highlighted in the editorial section of IEEE/AIP journal Computing in Science and Engineering (March-April 2003 issue).

Regulation of Cdk2-cyclin E activity. After fertilization, frog eggs undergo 12 rapid (30 min) synchronous divisions to generate a hollow ball of 4096 cells. During each cell division cycle, MPF (Cdk1-cyclin 


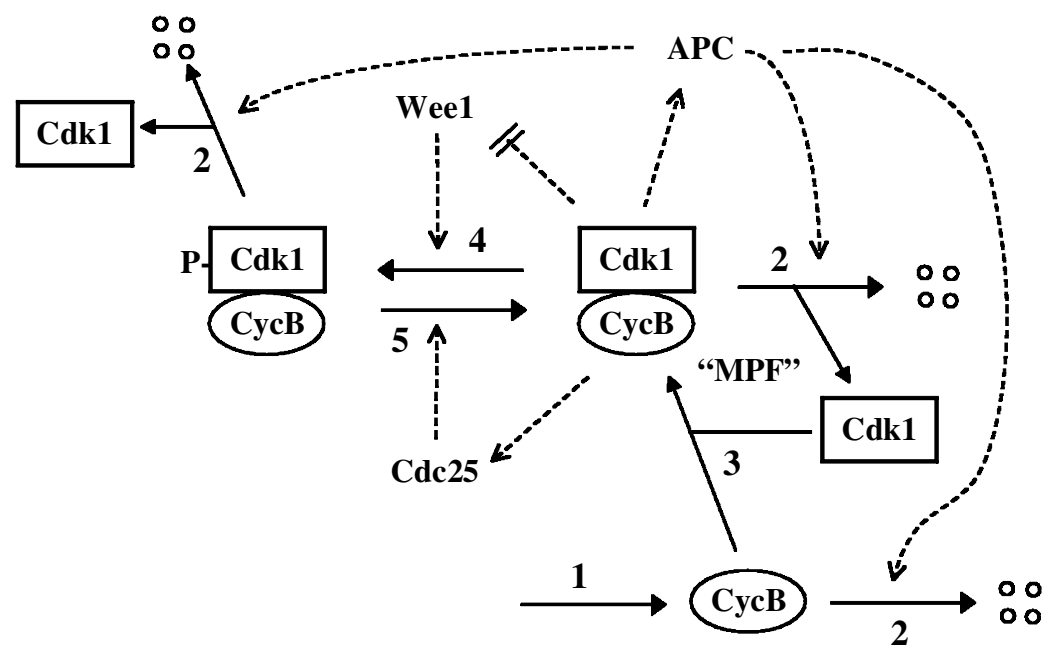

FIG. 1. Activation of M-phase promoting factor (MPF) in frog eggs (Novak and Tyson, 1993). Mitosis is initiated by cyclin-dependentkinase (Cdk1) in association with cyclin B (CycB). In frog eggs, CycB is synthesized steadily (reaction 1) and degraded periodically (reaction 2) by the anaphase promoting complex, APC (the four small circles represent products of cyclin degradation). $\mathrm{CycB}$ combines rapidly with Cdk1 subunits (reaction 3) to form active MPF. MPF is inactivated by phosphorylation (reaction 4; catalyzed by Wee1) and re-activated by dephosphorylation (reaction 5; catalyzed by Cdc25). Three feedback signals control this network: MPF activates Cdc25 (positive feedback), MPF inactivates Wee1 (mutual antagonism), and MPF activates the APC (negative feedback).

B) fluctuates from low activity (interphase) to high activity (metaphase), correlating with periodic degradation of cyclin B. For each cycle of MPF activity, the dimer Cdk2-cyclin E (an initiator of DNA synthesis in metazoans) undergoes two peaks of activity (15-min period), with highs in interphase (when DNA is being synthesized) as well as mitosis (Hartley et al., 1996, 1997). Whereas Cdk1-cyclin B oscillations are driven by periodic bursts of cyclin B degradation (Murray and Kirschner, 1989), Cdk2-cyclin E activity fluctuates in spite of constant levels of both Cdk2 (Howe and Newport, 1996) and cyclin E (Hartley et al., 1996). After cycle 12, the egg's pool of cyclin E is abruptly degraded (Hartley et al., 1996), by an unknown mechanism independent of cell number, protein synthesis and MPF activity.

We have proposed a molecular mechanism and mathematical model for these curious features of Cdk2cyclin E activity in early frog embryos (Ciliberto et al., 2003b). We assume that (i) Cdk2-cyclin E oscillations are driven by periodic inhibitory phosphorylations of the Cdk2 subunit by Wee1 kinase, and (ii) cyclin $\mathrm{E}$ degradation is dependent on autocatalytic loading of Cdk2-cyclin E onto a nuclear structure. We have tested some predictions of the model. For instance, when the embryo is injected with the recombinant protein Xic1 $\Delta 34$, this specific inhibitor of Cdk2-cyclin E activity blocks the 15-min oscillations, as expected, and delays the degradation of cyclin $\mathrm{E}$ until a few hours after Xic1 $\Delta 34$ itself is degraded. The model predicts that Cdk2-cyclin E and Wee1 are involved in a negative feedback loop, in contrast to Cdk1-cyclin B and Wee1, which are mutual antagonists. Direct experimental evidence for the negative feedback loop, which has yet to be obtained, will provide a critical test for this model.

\section{Budding yeast}

The basic cell cycle engine. The molecular machinery of the eukaryotic cell cycle control is known in more detail for budding yeast, Saccharomyces cerevisiae, than for any other organism (Mendenhall and Hodge, 1998). Molecular biologists have painstakingly dissected and characterized individual components and their interactions to derive a consensus picture of the regulatory network (Fig. 3). Some years ago, Chen et al. (2000) published a thorough computational exploration of this model, and recently Chen and Calzone (in preparation) have extended the model considerably to include the complex interactions of proteins con- 


\section{ALLEN ET AL.}

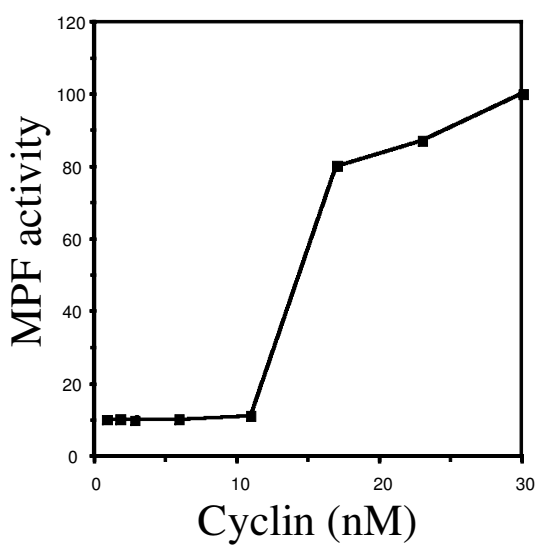

(a)

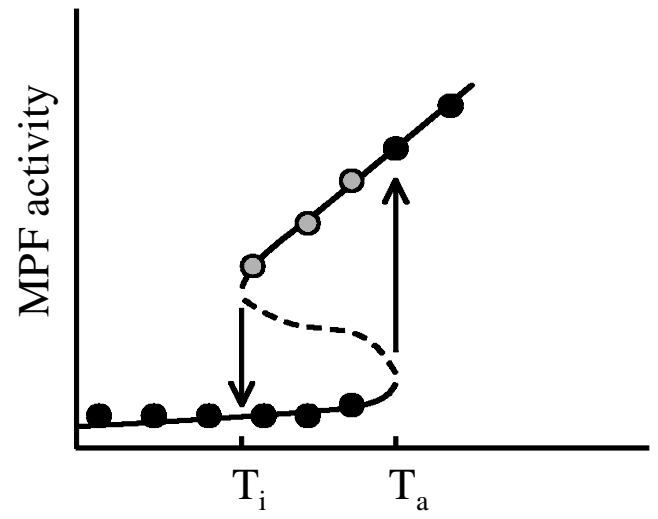

Cyclin

(b)

(c) Cyclin concentration increasing

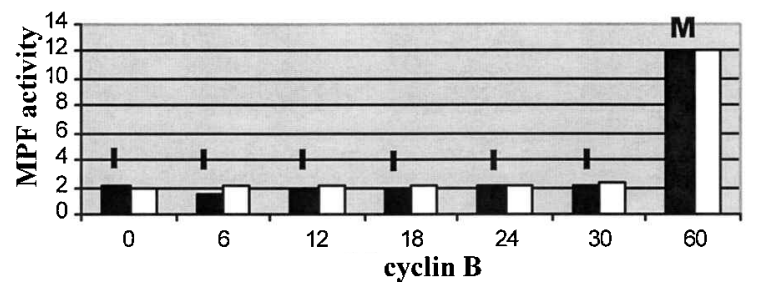

(d) Cyclin concentration decreasing

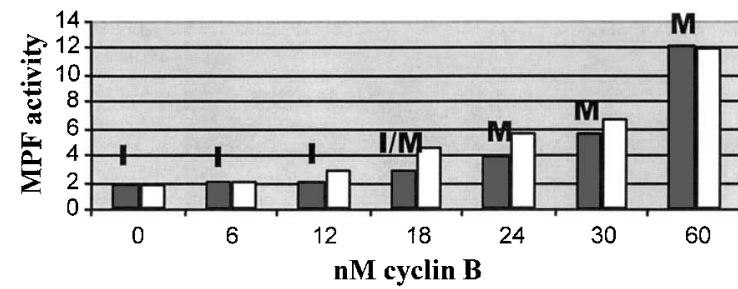

\section{Metaphase}

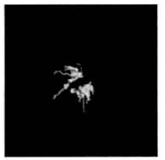

Interphase
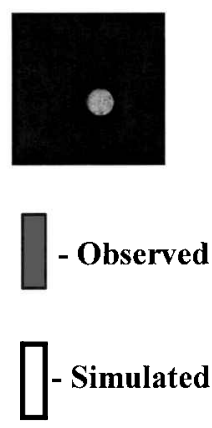

FIG. 2. Bistability of MPF activation in frog eggs. (a) Solomon et al. (1990) measured MPF activity in frog egg extracts supplemented with a fixed amount of non-degradable cyclin B, observing a characteristic cyclin threshold for MPF activation. (b) Novak and Tyson (1993) predicted that Solomon's threshold is indicative of an S-shaped dependence of MPF activity on cyclin level. For cyclin concentrations between the two thresholds, the control system has two stable steady states (interphase and M phase) separated by an unstable steady state (---). (c,d) Sha et al. (2003) measured MPF activity in extracts supplemented with increasing and decreasing amounts of cyclin B. "I" and "M" refer to the morphology of nuclei within an extract (interphase and metaphase, respectively; see insets). With increasing cyclin B concentration (panel c) Sha et al. confirmed Solomon's threshold. With decreasing cyclin B concentration (panel d) they confirmed the predictions of Novak and Tyson: (i) the cyclin threshold for MPF inactivation is about three times lower than the cyclin threshold for MPF activation, (ii) the control system is bistable for cyclin concentrations between the thresholds, (iii) MPF activity on the upper branch of the S-shaped curve is roughly proportional to cyclin concentration. Sha et al. confirmed two other major predictions of the Novak-Tyson model, as described in the text. Reprinted from the Proceedings of the National Academy of Sciences, U.S.A., copyright (2003).

trolling exit from mitosis. The new model, developed in collaboration with Fred Cross, consists of about 35 differential and algebraic equations for the regulatory protein species and their complexes (some of which are indicated in Fig. 3). The governing equations contain more than 100 parameters (kinetic constants) that must be estimated. 


\section{MODELING REGULATORY NETWORKS AT VIRGINIA TECH}

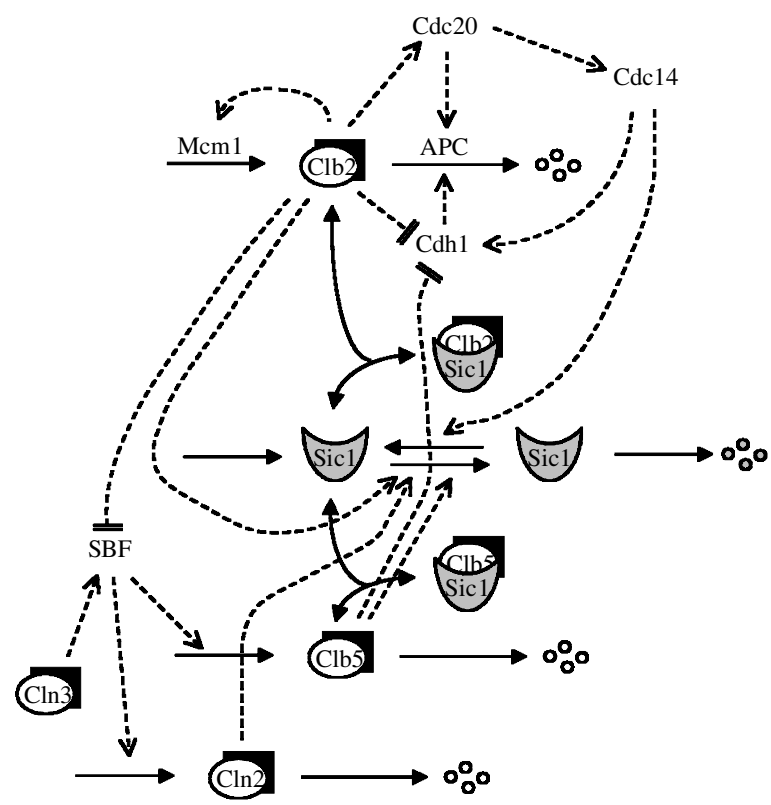

FIG. 3. Cell cycle control system in budding yeast (Chen et al., 2000). Cell cycle events in budding yeast are triggered by Cdk1 (black rectangle) in association with a variety of cyclins: Cln2 (primarily responsible for bud emergence), Clb5 (DNA synthesis), and Clb2 (mitosis). Cyclin levels are controlled by synthesis (transcription factors are SBF and Mcm1), by degradation, and by a stoichiometric inhibitor (Sic1). (The binding of Cdk1 to cyclin and the release of Cdk1 upon cyclin degradation are not represented explicitly on this diagram.) Clb2 degradation by the anaphase promoting complex (APC) is mediated by $\mathrm{Cdc} 20$ and $\mathrm{Cdh} 1$. The many feedback signals in the control system are indicated by dashed lines (catalytic actions). Especially noteworthy are (i) the mutual antagonism between the Clb-dependent kinases and their enemies (Sic1 and Cdh1), and (ii) the negative feedback loop whereby Cdk1-Clb2 activates Cdc20-APC activates Cdc14 activates Sic1 and Cdh1, which then inactivate Cdk1-Clb2.

The model has been verified in three specific ways. First of all, it must be consistent with the phenotypes of over 100 mutants that have been constructed and characterized experimentally by deleting or overexpressing the genes that code for all the components of the mechanism singly and in myriad combinations. To test for consistency, we define a "basal" parameter set, presumed to describe wild-type budding yeast cells. The solution of the governing equations with this parameter set must be consistent with the physiology of wild-type cells: for example, durations of the unbudded and budded phases of the cell cycle, size of the cell at the onset of DNA synthesis and at division, and the relative amounts of key regulators in the cell at different stages in the cycle. Next, for each mutant, we are allowed to change only certain parameters in specific directions. For instance, if the gene for $\mathrm{Cln} 2$ is deleted, then we must set the rate constant for Cln2 synthesis to zero. If the $\mathrm{Clb} 2$-gene is engineered to remove the amino acid sequence in $\mathrm{Clb} 2$-protein recognized by Cdc20 and Cdh1, then we must set to zero the rate constants characterizing Cdc20- and Cdh1dependent degradation of Clb2. All other parameters in the basal set must remain as they are. When the governing equations are solved with a "mutant" parameter set, the model must be consistent with the observed phenotype of that particular mutant. For example, the Cln2-deletion mutant is perfectly viable, but it is large and bud emergence is significantly delayed (Dirick et al., 1995). Over-expression of the Clb2degradation deficient gene renders cells inviable, blocked in late anaphase (chromosomes separated but cell undivided) (Amon et al., 1994).

Phenotypic details of the 125 mutants in our data set provide considerable constraints on the 100-dimensional parameter space, allowing us to estimate all the parameters in the model and to test the accuracy and sufficiency of the wiring diagram. These estimated values are predictions, and Mendenhall is developing methods for direct measurement of some of these rate constants on purified proteins. In addition, the 
model can be used to predict the phenotypes of specific mutants that have not yet been constructed. A number of predictions of the model about mitotic-exit mutants have been tested recently by Cross (2003), and they are confirmed.

The second test concerns the link between cell size and progress through the yeast cell cycle (Futcher, 1996; Johnston et al., 1977; Tyson et al., 1995). In the model, this link is forged by an assumption that Cdk1-cyclin dimers accumulate in the nucleus of the cell, achieving intra-nuclear concentrations that are proportional to total cell size (total number of ribosomes). Miller and Cross (2001) have tested this crucial assumption of the model by manipulating the "nuclear localization signals" on Cln proteins. By increasing or decreasing the targeting of Clns to the nucleus, Miller and Cross made cells smaller or larger, respectively, as predicted.

The third test concerns bistability in the budding yeast cell cycle control system (Fig. 4). As Chen et al. (2000) pointed out, the antagonism between the Clb-dependent kinases (Cdk1-Clb2 and Cdk1-Clb5) and their enemies (Sic1 and Cdh1) creates two, coexisting, stable steady states (Fig. 4a): a "G1" state (low activities of the $\mathrm{Clb}$-dependent kinases and high activities of their enemies) and a "S-G2-M" state (vice versa). "Helper" proteins, Cln2 and Cdc20-Cdc14 (Fig. 4a), drive transitions between these two states. Early in the cell cycle, rising activity of Cdk1-Cln2 destabilizes the G1 state and forces the transition to the S-G2-M state. Physiologists call this transition "Start." At the end of the cycle, activation of Cdc20 and Cdc14 destabilize the S-G2-M state and force the transition back to G1 (called "Finish" or "exit from mitosis"). Cross et al. (2002) tested this prediction of the model with an engineered strain of budding yeast that allowed them to control precisely the relative activities of Cln-dependent kinases and of Cdc14. They showed that, when both activities are zero, the control system could stably arrest in either G1 or M phase of the cell cycle, depending on which transition was last induced (Fig. 4b).

The morphogenetic checkpoint. Comparing Figures 1 and 3, we see a significant difference between frog and yeast cell cycle control: in frog eggs, a major role is played by Cdk1 phosphorylation-inactivation by Wee1 (and dephosphorylation by Cdc25), whereas in the budding yeast wiring diagram, the analogous reactions are absent. Indeed, budding yeast cells contain a kinase and phosphatase homologous to Wee1 and Cdc25 (Booher et al., 1993), but these enzymes seem to play no role in the normal cell cycle (Amon et al., 1992; Sorger and Murray, 1992). Cells with mutated Cdk1 that cannot be inactivated by Wee1 (CDC28AF) are perfectly viable in the presence of DNA synthesis inhibitors, and their "unreplicated DNA checkpoint" mechanism is still intact. Most eukaryotic cells are prevented from entering mitosis if there are problems in DNA replication, and this block is imposed by Cdk1 phosphorylation by Wee1. Not so in budding yeast, which enter mitosis just fine in the presence of unreplicated DNA, but cannot exit.

In budding yeast, Wee 1 and Cdc25 are used instead to monitor a "morphogenetic checkpoint" (Lew, 2000). If bud emergence is aborted (for example, by drugs or by mutations that block actin polymerization), entry into mitosis is delayed. This delay is achieved because Cdk1-Clb2 is inhibited by phosphorylation, catalyzed by a checkpoint-activated form of Wee1 (Sia et al., 1998). The delay prevents the formation of di-nucleate cells, which, though viable, are at a selective disadvantage to mono-nucleate cells (Sia et al., 1996).

The morphogenetic checkpoint is also intimately connected with a developmental switch in the life cycle of yeast, when they switch from the unicellular, budding mode of reproduction to a multicellular, pseudohyphal mode of growth (Edgington et al., 1999; Loeb et al., 1999). We have studied the wiring diagram for the morphogenetic checkpoint in great detail (Ciliberto et al., 2003a), comparing its behavior to the phenotypes of mutants studied in the laboratory of Daniel Lew at Duke University.

\section{Other modeling efforts}

Tyson's group is also involved in stochastic modeling (Mohsen Sabouri-Ghomi) and bifurcation analysis (Dorjsuren Battogtokh) of the budding yeast cell cycle, and in bifurcation analysis (Emery Conrad) and mechanisms of temperature compensation (Christian Hong) of circadian rhythms in fruit flies. Novak's group is modeling the fission yeast cell cycle (Akos Sveiczer), patterns of growth in fission yeast (Attila Csikasz-Nagy), and bistability in the signaling pathway for programmed cell death (Bela Gyorffy). 
$\mathbf{a}$
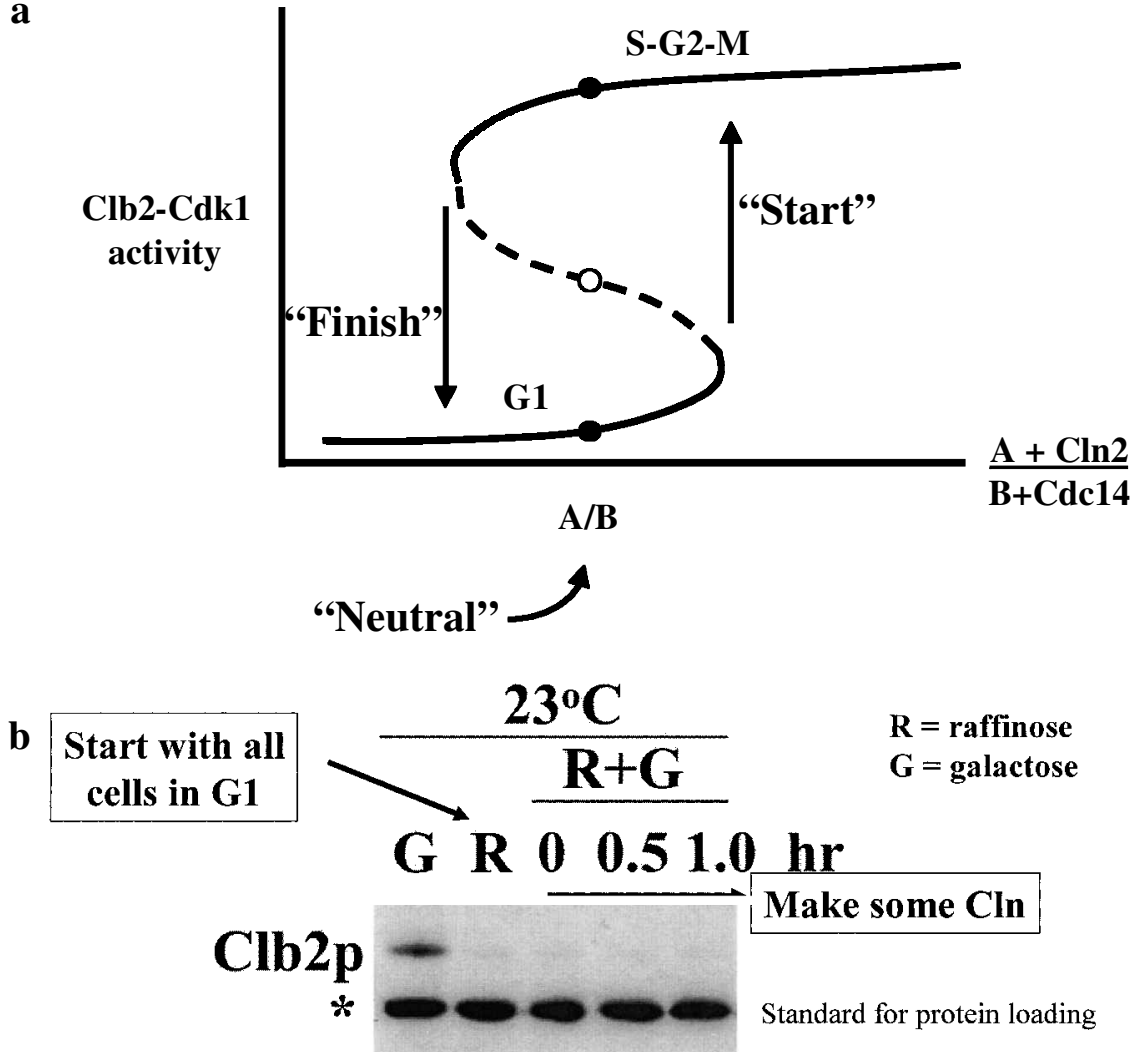

$88889146 \%$ Unbudded

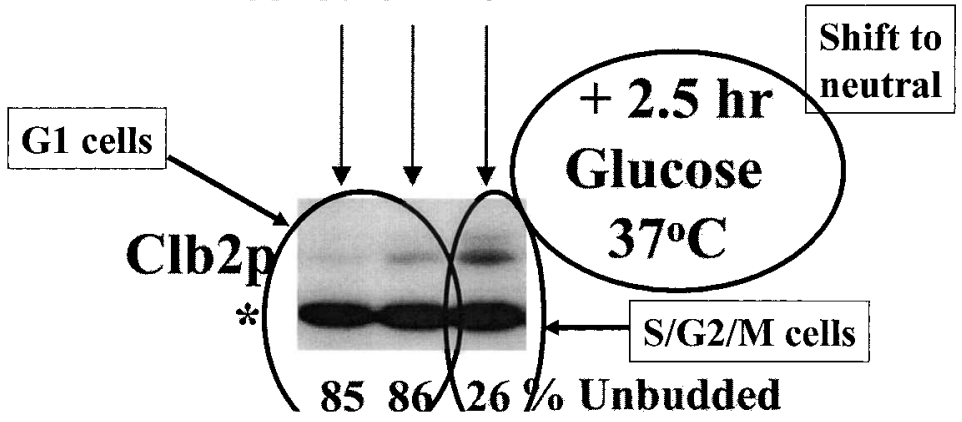

FIG. 4. Bistability in the budding yeast cell cycle. (a) Chen et al. (2000) predicted that the activity of Cdk1-Clb2 should show an S-shaped dependence on the combined activities of the Start-promoters (Cln2- and Cln3-dependent kinases) and the Finish-promoters (Cdc20 and Cdc14). When the promoters are absent, the control system is in the "neutral" position (A/B in the figure) and may persist indefinitely in either G1 phase (low Clb2-dependent kinase activity) or M phase (high Clb2 activity). (b) Cross et al. (2002) tested the prediction in a yeast strain allowing them to control Cln3 expression by galactose induction and Cdc14 activity by temperature. "Neutral" corresponds to cells growing on glucose (Cln3 synthesis off) at $37 \mathrm{C}$ (Cdc14 inactive). Cell cycle phase is monitored by budding index and Clb2 level (G1 = low budding index and $\mathrm{Clb} 2$ absent, $\mathrm{M}=$ high budding index and $\mathrm{Clb} 2$ present). The figure shows that cells in neutral can arrest stably in either G1 or M phase, depending on which transition (Start or Finish) was the last event experienced. Reprinted from Molecular Biology of the Cell (2002, vol. 13, pp. 52-70), with permission by the American Society for Cell Biology.

\section{SOFTWARE DEVELOPMENT}

JigCell refers to our suite of software components intended to provide a problem-solving environment (PSE) (Ramakrishnan et al., 2002) for modeling molecular regulatory networks. JigCell was developed in 
close collaboration with the modeling team using standard participatory design techniques. Rather than basing it on existing, general-purpose PSEs, JigCell was tailored to the specific needs of molecular cell biologists. This approach has not been a significant drawback, since most of the development work relates to domain-specific support rather than modeling infrastructure. We intend to support experts in molecular biology and biophysical chemistry who do not have significant experience in formal modeling. As much as possible, we employ off-the-shelf components, such as libraries of numerical algorithms, visualization tools, and communication protocols, where quality implementations exist and technical specifics can be hidden from the user.

In order to support the "modeling cycle" (Fig. 5) exemplified in the previous section, JigCell has three major components. The Model Builder assists the user to translate a wiring diagram into a correct and consistent set of differential-algebraic-discrete equations. The kinetic constants in the model, which are for the most part unknown at this stage, are given names instead of numerical values. The Run Manager associates a particular experimental protocol with a model (mathematical equations), a specific set of parameter values and initial conditions, and specifications for numerical simulation of the equations. The Run Manager tracks instructions for deriving the specific parameter values from a "basal" parameter set, so that the modeler can easily explore the dynamics of a model by twiddling the basal parameter values. The Comparator contains experimental data, a pointer to the appropriate row in a run file for simulating this data, output from the simulation, instructions for transforming simulation results into predictions in the same format as the experimental data, and a facility for quantifying the goodness-of-fit of the simulated to observed data and flagging outliers.

\section{Model builder}

The Model Builder (Vass et al., 2003) assists the user in specifying reaction equations and kinetic information of a molecular regulatory network. The information is displayed in spreadsheet format. A row of

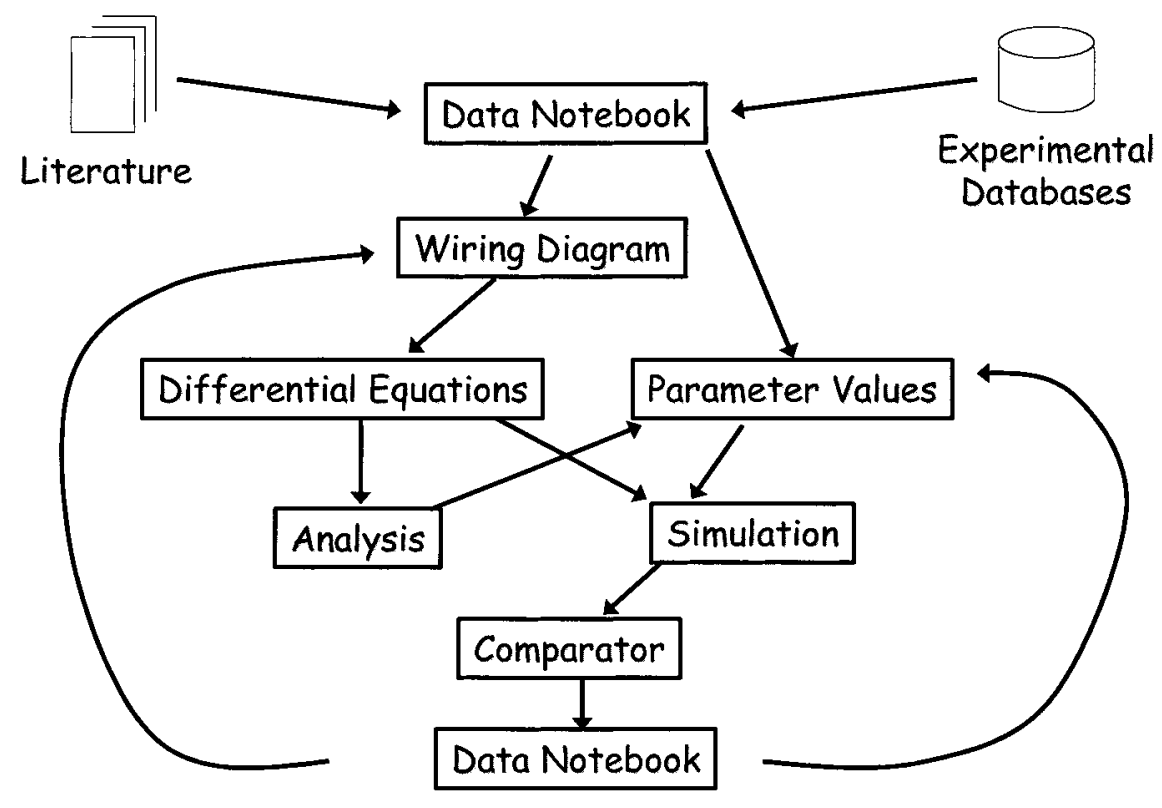

FIG. 5. The modeling cycle. The modeler starts with a hypothetical wiring diagram that he or she thinks is consistent with a set of experimental observations on a certain aspect of cell physiology. To test this hypothesis, the wiring diagram must be translated into a set of dynamical equations, parameters in the equations must be estimated, simulations run, and the model output compared to the original experimental data. Typically the output looks promising but is not in good quantitative agreement with experiment. Discrepancies trigger the inner loop of parameter adjustments to get a better fit. If no amount of parameter twiddling can bring the model in alignment with the data, then the modeler must consider changes in the wiring diagram itself, which starts the whole process from the top. 


\section{MODELING REGULATORY NETWORKS AT VIRGINIA TECH}

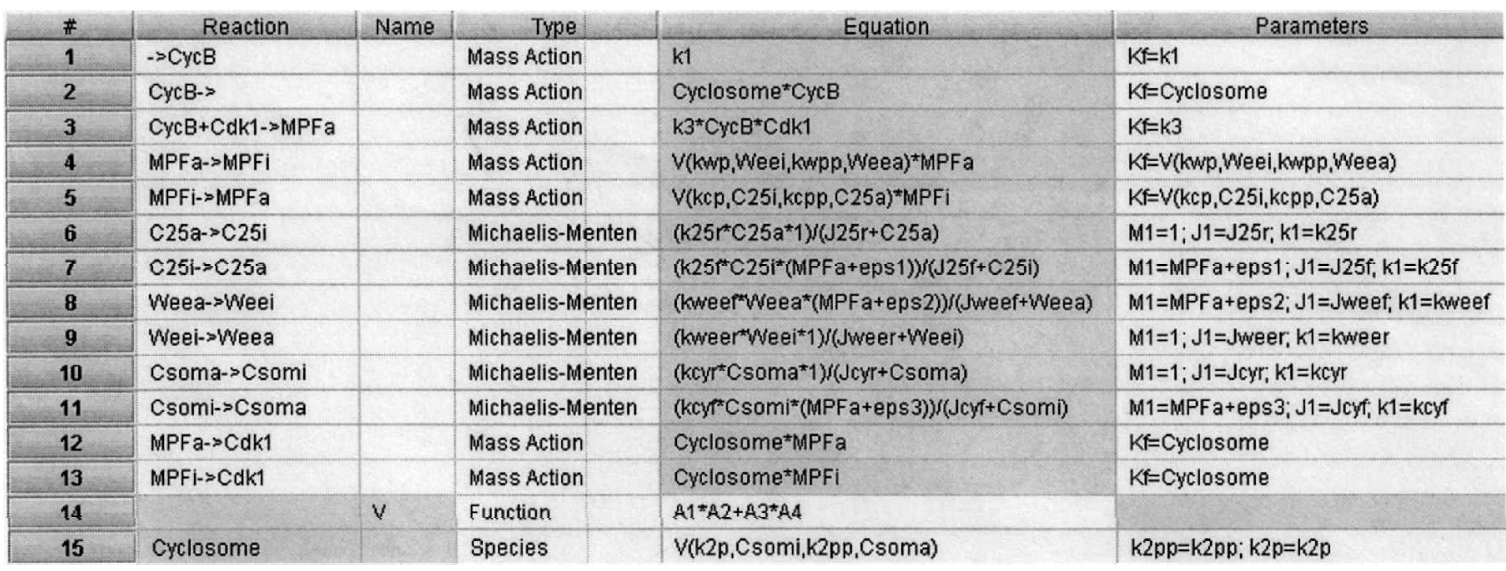

FIG. 6. The Model Builder. An implementation of the frog egg model in Figure 1. The APC is sometimes called the Cyclosome, hence the variable "csome" in this implementation.

the spreadsheet specifies a single chemical reaction: its substrates and products, stoichiometry, kinetic rate law, enzymatic catalysts, and designations of rate constants. For example, if we were working on the wiring diagram in Figure 1, we might write for reaction 4 something like this: Cdk1CycB is reset to PCdk1CycB, Michaelis-Menten kinetics, enzyme = Wee1, turnover number $=$ "kwee", Michaelis constant $=$ "Kmwee." (The precise syntax of the Model Builder is illustrated in Fig. 6.) Chemical reactions and rate laws provide a natural, precise, and concise representation of molecular regulatory networks of the type envisioned by BioSPICE. The Model Builder can also handle discrete events, such as cell division [mass $(\mathrm{t}) \rightarrow$ mass $(\mathrm{t}) / 2$ ], when a certain condition is fulfilled (e.g., Clb2(t) drops below a given threshold value).

Species names and kinetic information are checked continuously during model entry, with color highlights indicating portions of the model that are not correctly specified. The Model Builder also checks continuously for conserved quantities in the mechanism. For example, if the only reactions involving Wee1 are inter-conversion between active and inactive forms, then Wee1_total = Wee1_active + Wee1_inactive is a conserved quantity (a constant). In networks of even modest complexity, it is difficult to identify and implement these conservation conditions correctly. The Model Builder finds them automatically from the matrix of stoichiometric constants.

When a wiring diagram is fully entered into the spreadsheet and all inconsistencies have been resolved, the Model Builder constructs the governing equations and outputs them in three formats (at present). Because SBML (Systems Biology Markup Language) is becoming the standard interchange language in the modeling community, the Model Builder reads and writes models in SBML Level 2 (www.sbwsbml.org/sbml/docs/index.html). Because the Tyson lab uses PhasePlane and LSODE as its standard simulator packages, the Model Builder also writes the differential-algebraic-discrete equations as PhasePlanespecific ".ode" files and generic FORTRAN subroutines.

The Model Builder will be able to support stochastic models when it is integrated with BIONETS, a stochastic simulator package being developed by another BioSPICE group (led by Tim Elston). Spatial modeling, at present, is supported only as subdivision of a cell into topologically distinct compartments. Part of the problem here is that the modeling community has not yet settled on a general framework for stochastic and spatial modeling (in terms of SBML standard syntax).

\section{Run manager}

Also in spreadsheet format, the Run Manager (Fig. 7) is intended to describe an ensemble of simulation runs. Each row specifies how to simulate a certain experiment: the model, the parameter values and initial conditions, and the simulator to use. For instance, suppose we wanted to describe the simulation of a series of yeast cell mutants. The first row of the Run Manager might describe how to simulate wild-type cells, using a basal parameter set. The second row might describe the simulation of a Cln2-deletion mutant us- 


\section{ALLEN ET AL.}

\begin{tabular}{|c|c|c|c|c|c|c|}
\hline \# & Name & Comments & Model & Parameters & Initial Conditions & Simulator Parameters \\
\hline 1 & C.2 & J. Moore Timelags for MPF activation & frogegg.sbml & TotalCyclin.2 & Mi.2 & xpp50 \\
\hline 2 & C.25 & J. Moore Timelags for MPF activation & frogegg.sbml & TotalCyclin. 25 & Mil.25 & xpp50 \\
\hline 3 & C. 3 & J. Moore Timelags for MPF activation & frogegg.sbml & TotalCyclin.3 & Mi.3 & xpp50 \\
\hline 4 & C.5 & J. Moore Timelags for MPF activation & frogegg.sbml & TotalCyclin.5 & Mi.5 & крp50 \\
\hline 5 & Threshold1 & J. Moore Thresholds for MPF activation and inactivat... & frogegg.sbml & FrogEggBasalParams & FrogEggBasallCs & xpp50 \\
\hline 6 & Threshold2 & J. Moore Thresholds for MPF activation and inactivat... & frogegg.sbml & FrogEggBasalParams & FrogEggBasallCs & xpp50 \\
\hline 7 & Kumagai1 & Kumagai \& Dumphy 1995 Fig 4b Timecourse data f... & frogegg.sbml & FrogEggBasalParams & M1C1MO & xpp50 \\
\hline 8 & Kumagai2 & Kumagai \& Dumphy 1995 Fig 4b Timecourse data f... & frogegg.sbml & FrogEggBasalParams & FrogEggBasallCs & xpp50 \\
\hline 9 & Kumagai3 & Kumagai \& Dumphy 1995 Fig 3c Timecourse data f... & frogegg.sbml & FrogEggBasalParams & M1C1W0 & xpp50 \\
\hline 10 & Kumagai4 & Kumagai \& Dumphy $1995 \mathrm{Fig} 3 \mathrm{c}$ Timecourse data f... & frogegg.sbml & FrogEggBasalParams & FrogEggBasallCs & xpp50 \\
\hline 11 & Kumagai5 & Kumagai \& Dumphy 1992 Fig $10 \mathrm{Cdc} 25$ timecourse... & frogegg.sbml & $\mathrm{Cdc} 25.54$ & M.25cowo & xpp50 \\
\hline 12 & Kumagai6 & Kumagai \& Dumphy 1992 Fig 10 Cdc 25 timecourse... & frogegg.sbml & Cdc25.54TotalCyclin.0 & Moc1Wo & xpp50 \\
\hline 13 & Tang & Tang Coleman Dumphy 1993 Fig 2 Wee 1 timecour... & frogegg.sbml & TotalCyclin.254 & M.254C1W1 & xpp50 \\
\hline 14 & Tang2 & Tang Coleman Dumphy 1993 Fig 2 Wee1 timecour... & frogegg.sbml & TotalCyclin.0 & MoC1W0 & xpp50 \\
\hline
\end{tabular}

FIG. 7. The Run Manager. Instructions for simulating a few experiments from Kumagai and Dunphy (1995).

ing exactly the same information as in row 1, except that the rate constant for synthesis of Cln2 (given the name "kscln2" in the Model Builder) is singled out and given the value 0. Suppose row 3 describes a mutant cell with two copies of the wild-type $C \ln 2$ gene. In this case, kscln 2 must be singled out and multiplied by 2. Now, if the modeler decides that his/her first guess of a numerical value for kscln2 is inopportune, it is a simple matter to change the value of kscln2 in the basal parameter set, and this change will be propagated automatically by the Run Manager to all simulations of mutants involving changes in expression of Cln2. By organizing information in this way, the Run Manager allows the modeler to explore the parameter space easily over a complicated set of simulations.

\section{Comparator}

The Comparator (Fig. 8) is a tool for quantitative comparison of experimental data to model simulations. It is organized as a series of spreadsheet tabs. On the first tab, one enters experimental data as "lists of lists." Elements of these lists may be real numbers, integers, Boolean variables, or character strings. For instance, a row might contain the list (true, 1.8) whose elements answer the questions "is the mutant viable?" and "if so, how much larger is it at division compared to wild-type cells?" Or it might contain the list (false, "anaphase") whose elements answer the questions "is the mutant viable?" and "if not, at what phase in the cell cycle is it arrested?" A string like ((15.,0.06), (30.,0.13), (45.,30.7), . . ) might represent a series of measurements of MPF activity at different concentrations of total cyclin B (e.g., Solomon's [1990] experiment).

The second tab associates experimental data with simulation instructions on a row of a run file created by the Run Manager. Furthermore, once the simulation results are retrieved from the Run Manager, this tab

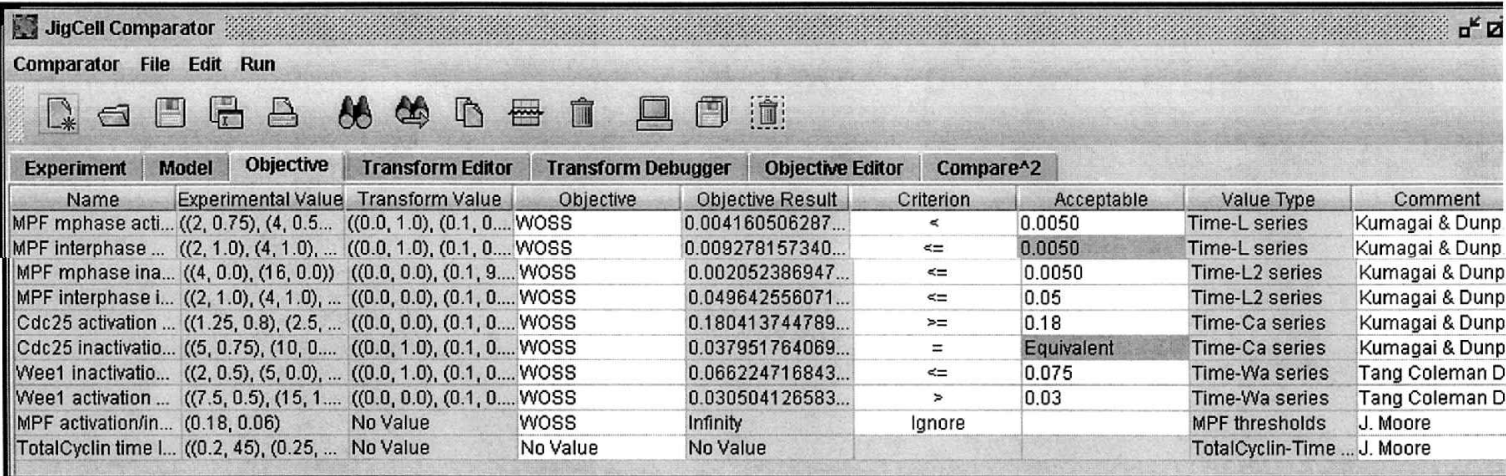

FIG. 8. The Comparator. Instructions for comparing the simulations in Figure 7 to the data in Kumagai and Dunphy (1995). 
contains a user-defined program for extracting the experimental observations, say (false, "anaphase"), from a table of numbers in the form $(t, x 1(t), x 2(t), \ldots)$.

The third tab describes how to compute a numerical value representing the goodness-of-fit of the simulation to the data, and specifies a threshold for this number, beyond which the fit is considered unacceptable. Unacceptable fits are highlighted in color so that the modeler can see at a glance where the model (equations plus parameter values) is having problems.

Exploration of parameter space, the inner loop of the modeling cycle (Fig. 5), is the most common and tedious chore in this business. By changing the numerical value of a suspect parameter in the basal set, the modeler can now see how the change propagates through the entire model and affects simulations of all the data to be modeled. The parameter change may indeed fix the problem for which it was intended, but may introduce new and unexpected discrepancies between the model and other experimental data.

By automating the comparison process, the Comparator takes all the drudgery out of the parameter-twiddling stage of model exploration, when the modeler is testing his/her intuition about the dynamic properties of a wiring diagram. It also provides the groundwork for the next stage: automatic parameter estimation. The computer can now score a fit of the model to the data and explore parameter space automatically for (possibly multiple) regions of good fit. Parameter optimization can be performed by local gradient-following algorithms or by global search algorithms. We are developing such tools, but they are not yet integrated into JigCell.

\section{Other components}

We are designing databases to manage the information to be generated by JigCell when it is fully operational (Dan Moisa, a graduate student in Ramakrishnan's lab). By recording usage of models and parameter sets and tracking simulations, run files, and comparisons as they are produced at every step of the modeling cycle, JigCell will enable better model management and facilitate efficient retrieval, searching, querying, and mining of the data.

We plan to use the BioSketchPad (http://bio.bbn.com/biospice/biosketchpad/index.html) as a graphical user interface for our Model Builder, and MONOD (www.molsci.org/Dispatch?action-WebdocWidget:4884detail=1) as our notebook for organizing the experimental information on which models are built. We are also developing tools to aid numerical bifurcation analysis of models.

In the future, it might be useful to borrow some ideas from the computer-game industry. Like heroes and villains fighting it out in a virtual castle, one might think of cell control systems as molecular agents (genes, proteins and metabolites) interacting according to fixed rules in a virtual environment resembling the internal structures of a eukaryotic cell. The biophysical chemistry underlying the rules must be solid, but approximations can be tolerated for the sake of computational speed and efficiency. Intriguing results can be confirmed using more accurate software in the BioSPICE toolkit. Game-based models with compelling graphics might be easily designed and sufficiently realistic and appealing to attract even the most reluctant molecular biologist to the modeling business.

\section{CONCLUSION}

Our experiences in modeling cell cycle regulation illustrate the challenges and promises of the BioSPICE program. With current software tools, a skilled modeler can create a realistic and accurate mathematical model of a complex molecular regulatory system, like that controlling cyclin-dependent kinase activity in eukaryotic cells. But the process is tedious and error prone, and not suitable for use by novices. Nonetheless, in the post-genomic world of molecular cell biology, there is a growing need for more and better models. To meet this need, the community requires software tools that will help experienced modelers to tackle problems of greater complexity and help novices to build correct and useful models of their latest laboratory experiments. In addition to BioSPICE, there are other recent attempts to provide the necessary computational modeling environment: Virtual Cell (Loew and Schaff, 2001), the Systems Biology Workbench (Hucka et al., 2003), GEPASI (www.gepasi.org), (Mendes, 1993; 1997), and E-cell (http://ecell.sourceforge.net/), (Tomita et al., 1999). At this stage, it seems wise to pursue a variety of ideas, keeping in mind 
that eventually the community will have to bring together the best ideas in a functional resource for the worldwide community of scientists interested in the molecular basis of cell physiology. To this end, the open-source philosophy of BioSPICE seems especially well suited.

\section{ACKNOWLEDGMENTS}

The work reported here has been supported primarily by the Defense Advanced Research Project Agency's Bio-Computation Program (AFRL F30602-02-0572), with additional support from the National Science Foundation (MCB-0078920 and MCB-0083315), the National Institutes of Health (R01-GM59688 and R01GM64339), the James S. McDonnell Foundation (21002050), and the Research Division of Virginia Tech. The views and conclusions contained herein are those of the authors and should not be interpreted as necessarily representing the official policies or endorsements, either expressed or implied, of the Defense Advanced Research Projects Agency (DARPA), the Air Force Research Laboratory, or the U.S. Government.

\section{REFERENCES}

AMON, A., IRNIGER, S., and NASMYTH, K. (1994). Closing the cell cycle circle in yeast: G2 cyclin proteolysis initiated at mitosis persists until the activation of G1 cyclins in the next cycle. Cell 77, 1037-1050.

AMON, A., SURANA, U., MUROFF, I., et al. (1992). Regulation of p34 ${ }^{C D C 28}$ tyrosine phosphorylation is not required for entry into mitosis in S. cerevisiae. Nature 355, 368-371.

BOOHER, R.N., DESHAIES, R.J., and KIRSCHNER, M.W. (1993). Properties of Saccharomyces cerevisiae wee1 and its differential regulation of $\mathrm{p} 34^{C D C 28}$ in response to $\mathrm{G} 1$ and $\mathrm{G} 2$ cyclins. EMBO J. 12, 3417-3426.

BRAY, D. (1995). Protein molecules as computational elements in living cells. Nature 376, 307-312.

CHEN, K.C., CSIKASZ-NAGY, A., GYORFFY, B., et al. (2000). Kinetic analysis of a molecular model of the budding yeast cell cycle. Mol. Biol. Cell 11, 369-391.

CILIBERTO, A., NOVAK, B., and TYSON, J.J. (2003a). Mathematical model of the morphogenesis checkpoint in budding yeast (Submitted to J. Cell Biol.; under revision).

CILIBERTO, A., PETRUS, M. J., TYSON, J.J., and SIBLE, J.C. (2003b). A kinetic model of the cyclin E/Cdk2 developmental timer in Xenopus laevis embryos. Biophys. Chem. 104, 573-589.

CROSS, F.R. (2003). Two redundant oscillatory mechanisms in the yeast cell cycle. Dev. Cell 4, 741-752.

CROSS, F.R., ARCHAMBAULT, V., MILLER, M., et al. (2002). Testing a mathematical model for the yeast cell cycle. Mol. Biol. Cell 13, 52-70.

DIRICK, L., BOHM, T., and NASMYTH, K. (1995). Roles and regulation of Cln/Cdc28 kinases at the start of the cell cycle of Saccharomyces cerevisiae. EMBO J. 14, 4803-4813.

EDGINGTON, N.P., BLACKETER, M.J., BIERWAGEN, T.A., et al. (1999). Control of Saccharomyces cerevisiae filamentous growth by cyclin-dependen kinase Cdc28. Mol. Cell. Biol. 19, 1369-1380.

FUTCHER, B. (1996). Cyclins and the wiring of the yeast cell cycle. Yeast 12, 1635-1646.

HARTLEY, R.S., REMPEL, R.E., and MALLER, J.L. (1996). In vivo regulation of the early embryonic cell cycle in Xenopus. Dev. Biol. 173, 408-419.

HARTLEY, R.S., SIBLE, J.C., LEWELLYN, A.L., et al. (1997). A role for cyclin E/Cdk2 in the timing of the midblastula transition in Xenopus embryos. Dev. Biol. 188, 312-321.

HOWE, J.A., and NEWPORT, J.W. (1996). A developmental timer regulates degradation of cyclin E1 at the midblastula transition during Xenopus embryogenesis. Proc. Natl. Acad. Sci. USA 93, 2060-2064.

HUCKA, M., FINNEY, A., SAURO, H.M., et al. (2003). The Systems Biology Markup Language (SBML): a medium for representation and exchange of biochemical network models. Bioinformatics 19, 524-531.

JOHNSTON, G.C., PRINGLE, J.R., and HARTWELL, L.H. (1977). Coordination of growth with cell division in the yeast Saccharomyces cerevisiae. Exp. Cell Res. 105, 79-98.

KUMAGAI, A., and DUNPHY, W.G. (1995). Control of the Cdc2/Cyclin B complex in Xenopus egg extracts arrested at a G2/M checkpoint with DNA synthesis inhibitors. Mol. Biol. Cell 6, 199-213.

LEE, T.H., TURCK, C., and KIRSCHNER, M.W. (1994). Inhibition of cdc2 activation by INH/PP2A. Mol. Biol. Cell. 5, 323-338.

LEW, D.J. (2000). Cell-cycle checkpoints that ensure coordination between nuclear and cytoplasmic events in Saccharomyces cerevisiae. Curr. Opin. Genet. Dev. 10, 47-53.

LOEB, J.D.J., KERENTSEVA, T.A., PAN, T., et al. (1999). Saccharomyces cerevisiae G1 cyclins are differentially 


\section{MODELING REGULATORY NETWORKS AT VIRGINIA TECH}

involved in invasive and pseudohyphal growth independent of the filamentation mitogen-activated protein kinase pathway. Genetics 153, 1535-1546.

LOEW, L., and SCHAFF, J.C. (2001). The Virtual Cell: a software environment for computational cell biology. Trends Biotechnol. 19, 401-406.

MARLOVITS, G., TYSON, C.J., NOVAK, B., et al. (1998). Modeling M-phase control in Xenopus oocyte extracts: the surveillance mechanism for unreplicated DNA. Biophys. Chem. 72, 169-184.

MENDENHALL, M.D., and HODGE, A.E. (1998). Regulation of Cdc28 cyclin-dependent protein kinase activity during the cell cycle of the yeast Saccharomyces cerevisiae. Microbiol. Mol. Biol. Rev. 62, 1191-1243.

MENDES, P. (1993). GEPASI: a software package for modelling the dynamics, steady states and control of biochemical and other systems. Comput. Appl. Biosci. 9, 563-571.

MENDES, P. (1997). Biochemistry by numbers: simulation of biochemical pathways with Gepasi 3. Trends Biochem. Sci. 22, 361-363.

MILLER, M.E., and CROSS, F.R. (2001). Mechanisms controlling subcellular localization of the G1 cyclins Cln2p and Cln3p in budding yeast. Mol. Cell. Biol. 21, 6292-6311.

MURRAY, A., and HUNT, T. (1993). The Cell Cycle. An Introduction (New York, W. H. Freeman \& Co.).

MURRAY, A.W., and KIRSCHNER, M.W. (1989). Cyclin synthesis drives the early embryonic cell cycle. Nature 339, 275-280.

NOVAK, B., and TYSON, J.J. (1993). Numerical analysis of a comprehensive model of M-phase control in Xenopus oocyte extracts and intact embryos. J. Cell Sci. 106, 1153-1168.

POMERENING, J.R., SONTAG, E.D., and FERRELL, JR., J.E. (2003). Building a cell cycle oscillator: hysteresis and bistability in the activation of Cdc2. Nat. Cell Biol. 5, 346-351.

RAMAKRISHNAN, N., WATSON, L.T., KAFURA, D.G., et al. (2002). Programing environments for multidisciplinary grid communities. Concurrency Comput. Practice Exp. 14, 1241-1273.

SHA, W., MOORE, J., CHEN, K., et al. (2003). Hysteresis drives cell-cycle transitions in Xenopus laevis egg extracts. Proc. Natl. Acad. Sci. USA 100, 975-980.

SIA, R.A., BARDES, E.S.G., and LEW, D.J. (1998). Control of Swe1p degradation by the morphogenesis checkpoint. EMBO J. 17, 6678-6688.

SIA, R.A., HERALD, H.A., and LEW, D.J. (1996). Cdc28 tyrosine phosphorylation and the morphogenesis checkpoint in budding yeast. Mol. Biol. Cell 7, 1657-1666.

SOLOMON, M.J., GLOTZER, M., LEE, T.H., et al. (1990). Cyclin activation of p34 ${ }^{\text {cdc2 }}$. Cell 63, 1013-1024.

SORGER, P.K., and MURRAY, A.W. (1992). S-phase feedback control in budding yeast independent of tyrosine phosphorylation of $\mathrm{p} 34^{\mathrm{cdc} 28}$. Nature $355,365-368$.

TOMITA, M., HASHIMOTO, K., TAKAHASHI, K., et al. (1999). E-CELL: software environment for whole cell simulation. Bioinformatics 15, 72-84.

TYSON, J.J., NOVAK, B., CHEN, K.C., et al. (1995). Checkpoints in the cell cycle from a modeler's perspective. In: Progress in Cell Cycle Research. L. MEIJER, S. GUIDET, and H.Y.L. TUNG, eds. (New York, Plenum Press), 1-8.

VASS, M.T., SHAFFER, C.A., RAMAKRISHNAN, N., et al. (2003). The Jigcell model builder: an error-reducing editor for creating regulatory network models. Bioinformatics (submitted).

WISE, S.M., SOMMESE, A.J., and WATSON, L.T. (2000). Algorithm 801:POLSYS_PLP: a partitioned linear product homotopy code for solving polynomial system equations. ACM Trans. Math. Software 26, 176-200.

ZWOLAK, J.W., TYSON, J.J., and WATSON, L.T. (2003). Parameter estimation for a mathematical model of the cell cycle in frog eggs. J. Comp. Biol. (Submitted).

Address reprint requests to:

Dr. John J. Tyson

Department of Biology (M.C. 0406)

Virginia Tech, Blacksburg VA 24061

E-mail: Tyson@vt.edu 